\title{
New Computing Model Helps Hamilton Health Sciences Address Changing Business Requirements
}

Mark Farrow

\begin{abstract}
This case study presents the impetus, business case, chronology and benefits of implementing a new server-based computing model at Hamilton Health Sciences that solved a critical desktop management problem while reducing IT costs. The new approach also provided a robust, flexible and scalable technology platform that is helping the hospital address business requirements driven by the emerging virtual healthcare community.
\end{abstract}

\section{Hamilton Health Sciences at a Glance}

Hamilton Health Sciences (HHS), which serves the more than 2.2 million residents of Hamilton, Central South and Central West Ontario, was formed through the merger of five hospitals and one cancer centre: Chedoke Hospital, Hamilton General Hospital, Henderson General Hospital, McMaster Children's Hospital, McMaster University Medical Centre, as well as the Juravinski Cancer Centre. Together, these facilities offer a range of acute and specialized services, catering to healthcare needs from preconception through to aging adults. This, in combination with its focus on academics and research, makes Hamilton Health Sciences the employer of choice for nearly 10,000 people. (www.hhsc.ca)

\section{Increasing Automation Strains IT Resources}

The adoption of technology at HHS is now in high gear as decision-makers begin to see its full potential as a critical enabler for reducing cost, improving clinical and operational efficiency, attracting and retaining the best medical professionals and improving patient care and safety.

As technology began to gain traction at HHS, however, the hospital's IT infrastructure and its Information \& Communications Technology team (ICT) began to feel the strain. Growing pressure from users for more and better applications was a big challenge, for example. ICT, a group of 80 people that was already managing more than 100 servers, 5,000 PCs and 10,000 users, was facing a queue of 177 application requests. Worse yet, the demand from users to keep up with the latest versions of PC applications such as the Microsoft Office Suite, presented an almost insurmountable problem, requiring the continual upgrading of application and operating system software in 5,000 PCs, a huge, time-consuming and expensive undertaking, even with the help of advanced tools. With the growing number of users and PCs, the ICT budget would not be able to sustain this operating model.

\section{Server-Based Computing Offers Significant Benefits}

Rather than throwing more people and money at the escalating desktop management problem, ICT decided to look for a new computing model that would enable the group to keep desktop systems current, reduce ongoing operating and support costs, address growing requirements for user mobility and quickly, easily and cost-effectively realign the hospital's IT infrastructure to meet rapidly changing business requirements, including emerging regional, provincial and national e-health initiatives.

A thorough analysis of the infrastructure supporting the hospital's current desktop implementation and a review of alternate approaches led to an investigation of how server-based computing (a.k.a. "thin-client computing") could help ICT achieve these objectives.

Server-based computing (SBC) simplifies the management and support of the desktop environment by moving applications and data off personal computers and onto corporate servers. While the look, feel and functionality of applications remains the same as perceived by the user, the user's PC becomes just a terminal device, passing keystrokes and mouse clicks up to, and displaying screen images sent down from, the applications running on the centralized corporate servers. 
The SBC computing model offers significant advantages. With virtually no processing to do, PCs need no application software and no hard disk drive (hence "thin client"), thus eliminating the need to "touch" every PC when a software upgrade is required or new application deployed - it's all done once, centrally on the application server(s). This significantly reduces desktop support complexity and cost, and makes application deployment, software upgrade and patch management quick, easy and inexpensive. With SBC, all users see the same version of applications, eliminating incompatibilities; and because the PCs require substantially less horsepower and memory, their useful lifetime can be significantly extended. In addition, "locking down" the PCs so that users can no longer load their own software greatly reduces support complexity.

\section{Pilot Test Results Support SBC Direction}

An evaluation of a number of SBC solutions by ICT during the summer of 2004 led to the selection of Citrix Presentation Server as the best alternative for HHS, both in terms of functionality and risk avoidance. In addition to having many large installations, including some within Canadian healthcare settings, Citrix technology is mature and is supported by a well-established network of certified resellers and specialists.

In early 2005, ICT conducted a pilot in the Burns Trauma Unit, where 30 users agreed to have their PCs locked down
In addition to providing the hospital's 10,000 users a consistent access experience, AccessHHS makes it easy for the hospital to extend its reach into the local and regional community the "virtual healthcare community."

Table 1. Financial projection (two-year rollout)

\begin{tabular}{|c|c|c|c|c|c|c|c|c|}
\hline Cost & Year 1 & Year 2 & Year 3 & Year 4 & Year 5 & Year 6 & Year 7 & Total \\
\hline Current Model & 900,000 & 900,000 & 900,000 & 900,000 & 900,000 & 900,000 & 900,000 & $6,300,000$ \\
\hline SBC Model & $1,000,988$ & 802,361 & 428,176 & 451,576 & 734,436 & 721,090 & 435,479 & $4,574,106$ \\
\hline Annual Savings & $(100,988)$ & 97,639 & 471,824 & 448,424 & 165,564 & 178,910 & 464,521 & $1,725,894$ \\
\hline Cumulative Savings & $(100,988)$ & $(3,349)$ & 468,475 & 916,899 & $1,082,463$ & $1,261,373$ & $1,725,894$ & $\$ 1,725,894$ \\
\hline Cash Flow & Year 1 & Year 2 & Year 3 & Year 4 & Year 5 & Year 6 & Year 7 & Total \\
\hline Current Model & 299,250 & 598,500 & 897,750 & $1,197,000$ & $1,197,000$ & $1,197,000$ & $1,197,000$ & $6,583,500$ \\
\hline SBC Model & 332,829 & 599,614 & 741,982 & 892,131 & 803,503 & 776,480 & 778,908 & $4,925,446$ \\
\hline Annual Savings & $(33,579)$ & $(1,114)$ & 155,768 & 304,869 & 393,497 & 420,520 & 418,092 & $1,658,054$ \\
\hline Cumulative Savings & $(33,579)$ & $(34,692)$ & 121,076 & 425,945 & 819,442 & $1,239,962$ & $1,658,054$ & $\$ 1,658,054$ \\
\hline
\end{tabular}


and turned into thin-client terminals and to access their applications solely using Citrix for a two-week period.

Overall, users were able to use the new interface to access their applications and perform their regular daily operations as expected. No functional issues were encountered during the test, with but one exception: a Glucometer application had to be installed on each PC and run locally, because the current release of that particular application would not run properly in a serverbased configuration.

As a result of what was learned from the pilot test, ICT management built a business case based on the recommendation that the hospital's current desktop environment be completely replaced by a new SBC model over a two-year period, using Citrix as the enabling technology. Although a lot of "what if" analyses were performed, ICT management chose a conservative scenario regarding hardware costs, staff reductions, savings

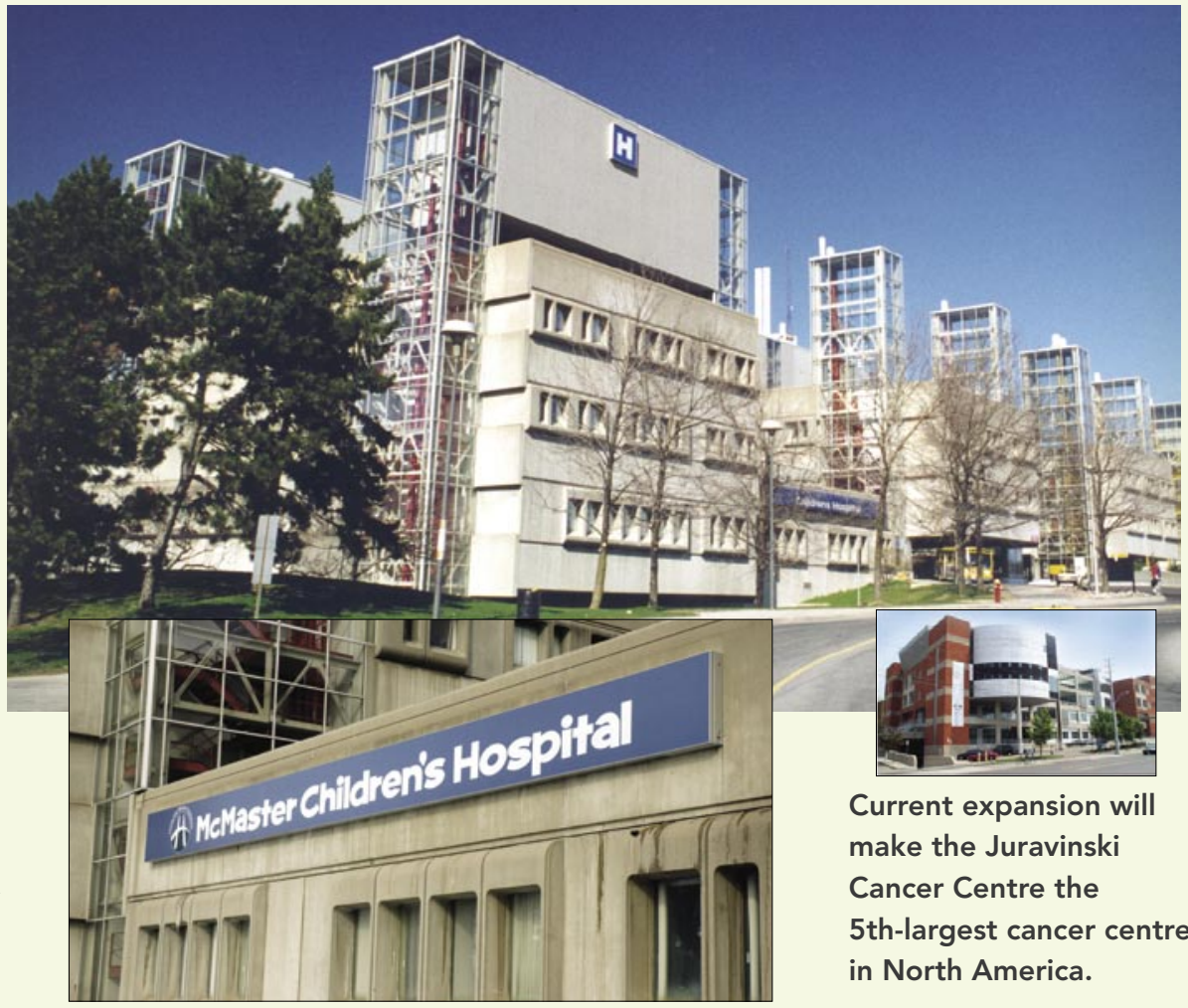

McMaster Children's Hospital is part of the McMaster University Medical Centre, home of Canada's most innovative Digestive Disease program. in computer room costs and a variety of other project metrics. Accordingly, the business case assumed that

- the project would be self-funded - new PCs and servers would be leased, for example, so they could be paid for out of savings in operating costs;

- only a modest reduction in staff requirements of 1.5 fulltime staff would be realized, and these would be reallocated to other priority ICT activities. Initial concern over possible job loss was averted through timely communications and showing staff how their jobs would in fact be enhanced;

- the simplified structure and reduced desktop management requirements of the SBC model would yield user-to-IT-staff ratios as high as 200:1, enabling ICT to increase its scope of activity and level of support without increasing its overall staffing complement for a number of years;

- only 3,500 user software licenses would need to be purchased (i.e., a maximum of 3,500 of the 5,000 total desktops would have an application session active concurrently);

- approximately 70 new application servers would be required (i.e., a user-to-server ratio of 50:1) to ensure adequate application performance;
- only $80 \%$ of the hospital's PCs would be able to be locked down into thin clients. The remaining $20 \%$ would need to stay "fat" for use by high-end power users and for special applications used in areas such as Finance;

- servers would be replaced every four years and client devices every six to seven years, a $50 \%$ increase in client device lifespan.

The business case that ICT presented to the hospital's Information Management Steering Committee showed a savings of \$1.6 million over seven years, with actual savings starting by year three. By year five, the savings would be greater than $\$ 500,000$ per year (see Table 1). Based on the projected savings and business benefits, the business case was approved, and the project moved forward into the final design phase.

\section{Strong Partnerships Key to Successful Project}

Following the success of the pilot, ICT contracted with Citrix to do the high-level, architectural design for a complete SBC solution that could be deployed to all HHS users. With a lot that had to be considered in order to do this project right, it was recommended that ICT also engage a knowledgeable IT services 
provider to help them. Accordingly, ICT sought out a services partner that not only had a good track record and experience with server-based computing, but was also aware of the unique issues confronting the healthcare industry.

After interviewing a number of prospective partners, ICT chose Compugen Inc., a national Citrix Platinum Solutions Advisor headquartered in Richmond Hill, Ontario, to help them finalize the design and implement their solution. In addition to having extensive SBC project experience, backed by solid customer references, Compugen was chosen because they have a substantial Citrix practice and are well plugged into the product direction.

\section{... the new approach is helping the hospital address business and cultural issues, and is providing a new computing model that is better able to meet current and future needs.}

With Compugen on board to help with requirements definition and vendor selection, HHS issued an RFP for the new hardware - primarily servers and storage - that would be needed to support an SBC implementation. Hewlett-Packard (HP) won the bid based on the strengths of the HP Blade System. Not only did the HP blade servers offer better price/performance than the competitive offering, their product roadmap meant that HHS's investment in server technology would be protected as new technologies emerged.

Systems management was also a key factor in choosing the HP hardware. HP's Systems Insight Manager (a set of software tools) would allow HHS system administrators to manage their entire SBC hardware infrastructure from a single console screen, enabling HHS to realize significant operational cost savings by having a unified systems management strategy that requires fewer ICT staff.

\section{Final Design Yields Additional Savings and Benefits}

In addition to what was learned from the pilot test, the final system design upon which the actual SBC deployment is based incorporated some innovative thinking by Hewlett-Packard and Compugen that resulted in some additional costs savings as well as a number of other technical and business benefits:
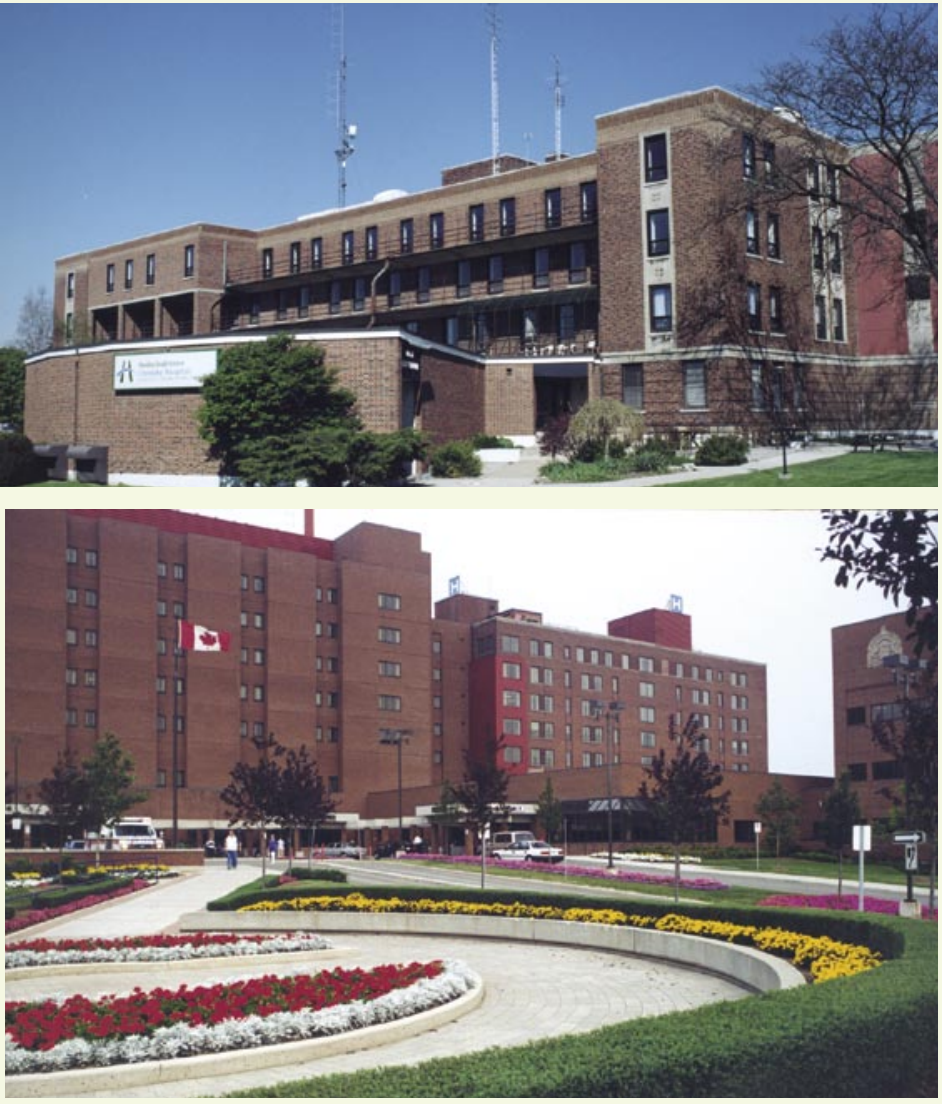

Chedoke Hospital hosts many of HHS's rehabilitation programs, while Henderson General boasts a highly regarded Orthopedic program.

Blade Servers - with HHS's infrastructure expected to grow to more than 100 servers, the use of blade servers is helping ICT reduce real estate requirements, drive down costs and simplify the environment needing support. The blade servers are easy to manage and can be quickly repurposed and redeployed to meet changing needs or fluctuations in HHS application workload.

Boot from SAN - Compugen and HP proposed the use of a fibre-attached Storage Area Network rather than each blade server having its own native disk drive. In addition to simplifying storage management, the use of a single, centralized SAN saves on storage costs, since fewer disk drives are required. Of even greater benefit, however, is the ability for the blade servers to be booted up from the SAN, making them easier to configure and manage, and bringing them closer to being "hot swappable" in the event of a failure.

64-Bit Computing - An analysis showed that a system design based on 64-bit computing would enable the application servers to support many more users. Although the HHS business case 
was initially based on 50 users per application server, the new 64-bit design that is actually being deployed is expected to support as many as 75 users per server, resulting in even better server utilization and reduced cost. Even though some of the hospital's older applications still require a 32-bit environment, HHS is one of the first organizations in Canada to be implementing 64-bit Citrix.

Disaster Recovery - Compugen and HP also proposed that the final system design include provision for disaster recovery at the two HHS datacentre sites - Hamilton General and McMaster Medical Centre - that HHS had not previously been able to do effectively due to budget limitations. By splitting its application server farm between the two sites and running mirrored applications - half the user sessions are hosted at one site and half at the other - ICT is now able to cost-effectively provision a basic disaster-recovery configuration. By also including a separate SAN at each site, one SAN can be replicated against the other and one SAN can be backed up to the other, providing additional redundancy and protection.

\section{Web Interface Addresses Key Usability Goal}

From the beginning, ICT has focused on creating a standardized environment for all users. A key aspect of this was the development of a Web-based user interface branded as "AccessHHS." Developed using Citrix's Web client, this portal-like interface (see Figure 1) is key to ensuring that users accessing hospital data or running applications all have a similar experience, regardless of where they connect from - within the hospital, another hospital, a clinic in the community, a doctor's office or from home.

Having users access applications through an Internet Explorer-like page using a standard Web browser ensures that the same interface is presented to an internal user attached to the internal network as it is to an external user who accesses the applications through an https-type Web site over the public Internet - the same type of secure site that is typically accessed for online banking, for example. In addition to providing the hospital's 10,000 users a consistent access experience, AccessHHS makes it easy for the hospital to extend its reach into the local and regional community - the "virtual healthcare community."

\section{SBC Platform Supports Evolving Business Requirements}

Expected to be fully complete by Fall 2006, the general deployment of the new SBC model at HHS is fully underway. In addition to solving rapidly growing problems associated with desktop management, support complexity and ongoing operating costs, and giving users consistent access to a common and current set of applications, the new approach is helping the hospital address business and cultural issues, and is providing a new computing model that is better able to meet current and future needs.

\section{Scaling Up with a Growing User Base}

The SBC solution, which combines thin clients, blade servers, SANs, 64-bit computing, Web-based access and single, centralized application deployment, is a highly flexible implementation model that will scale easily and cost-effectively as the hospital's user base grows. Additional blade servers and chassis are easy to install, for example, with little or no additional power, cabling or network connections required; and with 'drag \& drop' software imaging and configuration capability from a centralized console, administrators can have additional servers up and running in just minutes. With centralized application management, the desktop support model scales up with little or no additional staff required.

\section{Supporting a Highly Mobile Workforce}

With clinical staff on the go 24/7, the only way to truly increase hospital efficiency, improve patient care and safety, and to get maximum value out of professional staff is to make it as easy as possible for them to access hospital systems and patient information from wherever they are. ICT has been deploying wireless networks throughout HHS, for example; and with only keystrokes, mouse clicks and screen pixels traversing the network, SBC is ideally suited for the hospital's wireless environment, placing much less demand on network bandwidth and performance. This will allow HHS to improve patient care and safety by driving the "point of information access" closer to the bedside.

The new computing model also meets the requirements of hospital staff needing access to hospital resources from outside the hospital complex. For example, physicians may want to review diagnostic images or review and approve reports from home; or in areas such as transcription and coding, where recruiting has become more difficult, the ability to allow staff to work from home, where they have the flexibility to work around their families' needs and avoid unnecessary commute time and expense, has become a huge factor in being able to attract and retain qualified people. Using Citrix, HHS already has roughly 100 of its 120 coders and transcriptionists working from home, with the rest scheduled to work from home by year end.

\section{Connecting the Virtual Healthcare Community}

Looking at the Hamilton region from its perspective as a hub within LHIN 4 (one of 14 such organizations across the province that make up Ontario's new Local Health Integration Network structure), HHS sees a growing requirement to be able to communicate and share patient information with other members of the local healthcare community, including other 


\section{Happier clinical users and a more satisfied staff within ICT have made this project a success.}

hospitals, clinics, and mental health and long-term care facilities. The AccessHHS interface makes it easy for HHS to cooperate in this new community model by enabling any authorized user to quickly and easily access hospital information and applications from anywhere, via the public Internet using a standard Web browser.

On a broader scale, the SBC solution provides a flexible and robust platform upon which HHS can implement any information sharing requirements that may emerge from e-health initiatives at the provincial or federal level. SBC and AccessHHS provide a solid stepping stone for HHS to make information access and sharing outside the hospital walls a practical reality.

\section{Security and Regulatory Compliance}

As ICT expands the hospital's support for application mobility and remote access, the need to guard against the risk of theft or loss of PCs and laptops becomes a significant consideration. This is especially true in the context of the hospital's need to comply with Ontario legislation such as PHIPA (Personal Health Information Protection Act) governing the secure and confidential handling of personal patient information.

A number of security considerations are inherent in the SBC model. First and foremost, no applications or data reside on laptops or PCs, so private information is not at risk if client devices are stolen or lost. All user data sits on centralized storage within the secure HHS datacentres, where it is backed up regularly in accordance with best practices governing data protection. Although external access to HHS applications is via the public Internet, it is done using a secure, 128-bit encrypted connection, thus eliminating any concern that those connections can be intercepted. With data and applications all managed centrally, ICT controls who has access to what resources, and knows exactly who is accessing those resources at any given time.

\section{Pandemic Readiness}

The World Health Organization is now warning that a global Avian Flu crisis is inevitable, and possibly imminent. With many employees likely staying home for an extended period during a pandemic, it is being recommended that organizations examine their business processes and workflow to better understand how they can be executed in a virtual work environment. Since remote access is a key component of implementing a virtual work environment, the SBC solution gives $\mathrm{HHS}$ a head start on its pandemic readiness planning.

\section{Conclusion}

Without a massive infusion of additional money and staff, the hospital's traditional approach to managing computer users simply wasn't going to meet the technical and business requirements associated with operating an efficient clinical environment. By leveraging the strengths of its technology partners, HHS has been able to implement a totally new computing paradigm that has solved an escalating desktop management problem, while allowing the IT department to continue operating effectively within the boundaries of its fiscal realities. In SBC, HHS found a technology that is also enabling the hospital to address a number of unique business requirements stemming from the rapidly changing healthcare environment within the Hamilton and Niagara region. Happier clinical users and a more satisfied staff within ICT have made this project a success.

\section{About the Author}

Mark Farrow, Director, Information \& Communications Technologies, Hamilton Health Sciences, oversees a \$14 million operating budget and a staff of over 80 . With a Bachelor of Commerce in Finance and an M.B.A. in Information Management from McMaster University, Mark has a 20-year career in Information Technology and management, including his previous position as director of information systems at The Credit Valley Hospital in Mississauga, Ontario, where he implemented one of the first fully electronic patient records systems utilizing integrated hospital information systems and document imaging technologies.

\section{Join us at Compugen iForum North}

IT leaders in Healthcare and Government showcase their successful, leading Citrix implementations and their visions for the future.

\section{FEATURED SPEAKER:}

Mark Farrow, Director, Information \& Communications Technologies, Hamilton Health Sciences

November 23, 2006

InterContinental Toronto Centre

To register: www.compugen.com/seminar

CiTRIX COMPUGÉN 